Research

\title{
The clinicopathological characteristics, prognosis and immune microenvironment mapping in MSI-H/MMR-D endometrial carcinomas
}

\author{
Yu-e Guo ${ }^{1}$ Yin Liu ${ }^{2} \cdot$ Wei Zhang ${ }^{1} \cdot$ Heng Luo ${ }^{1} \cdot$ Ping Shu $^{1} \cdot$ Guofang Chen ${ }^{3} \cdot$ Yuping Li $^{1}$
}

Received: 30 September 2021 / Accepted: 5 January 2022

Published online: 03 March 2022

(c) The Author(s) 2022 OPEN

\begin{abstract}
Endometrial cancer had a relatively high prevalence of MMR deficiency. MMR-D/MSI-H endometrial cancer patients are suggested to be potential beneficiaries of PD-1/PD-L1 inhibitor therapy. Here, we explored the prognostic value of MSI subtype in endometrial cancer and its correlation with immune environment. Based on expression and clinical data of 78 POLE, $123 \mathrm{MSI}$ and 299 Other EC samples from the TCGA-UCEC project, we found that the MSI tumors were identified more often in early stage, had a lower age, better patient survival, enriched $C D 8^{+} T$ cells, and regulatory $T$ cells and less M2 macrophages and activated dendritic cells than the Other group, and shared a relatively similar expression profile with POLE group by differential analysis. In addition, we established the immune landscape of an MMR-D endometrial cancer tissue using unbiased single-cell RNA-seq analysis of 3371 cells. By immunohistochemistry analysis, we found that the MMR-D tumors showed a higher trend of $C D 20^{+} B$ cells infiltration. Our study might expand our understanding of the role of immune subsets in MSI endometrial carcinomas and provide guidance of immunotherapy for endometrial cancer.
\end{abstract}

Keywords Microsatellite instability (MSI) - Mismatch repair (MMR) - Endometrial carcinoma · Immune infiltration · Single-cell RNA-seq analysis

\author{
Abbreviations \\ EC Endometrial cancer \\ ECC Endometrioid carcinoma \\ MMR Mismatch repair \\ PTEN Phosphate and tension homology deleted on chromosome ten \\ POLE DNA polymerase $\varepsilon$ \\ BMI Body Mass Index
}

Yu-e Guo and Yin Liu contributed equally to this work

Supplementary Information The online version contains supplementary material available at https://doi.org/10.1007/s12672-02200466-5.

Guofang Chen, chenguofang@tongji.edu.cn; $\bowtie$ Yuping Li, gracelyp@163.com |'Department of Pharmacy, Shanghai Pulmonary Hospital, Tongji University School of Medicine, 507 Zhengmin Road, Shanghai 200433, China. ${ }^{2}$ Department of Laboratory Medicine, Shanghai Pulmonary Hospital, Tongji University School of Medicine, Shanghai, China. ${ }^{3} \mathrm{Clinical}$ and Translational Research Center, Shanghai First Maternity and Infant Hospital, Tongji University School of Medicine, 2699 West Gaoke Road, Shanghai 201204, China.

Discover Oncology $\quad$ (2022) $13: 12 \quad$ https://doi.org/10.1007/s12672-022-00466-5 


\section{Introduction}

Endometrial carcinomas (ECs) have been broadly categorized into two subtypes according to clinicopathologic characteristics, hormone receptor expression and grade in the past 30 years [1]. Type I tumors account for $70 \%$ to $80 \%$ of ECs, and are endometrioid, hormone-receptor-positive, low-grade ECs with a good prognosis. Type II ECs are non-endometrioid, hormone-receptor-negative, high-grade tumors, which exhibit higher risk of metastasis and poor outcomes [1,2]. However, the prognostic value of this dualistic classification remains limited, proper subtyping is critical for selecting appropriate treatment [1, 3]. In 2013, molecular classification by The Cancer Genome Atlas characterized endometrioid and serous endometrial cancer into four distinct molecular subgroups: POLE ultramutated, microsatellite instability hypermutated (MSI-H), copy number low (endometrioid) and copy number high (serous-like) [4]. Molecular classification is one of the most important developments in the study of endometrial carcinoma in recent years. The ESGO/ESTRO/ESP (the European Society of Gynaecological Oncology (ESGO), the European Society for Radiotherapy \& Oncology (ESTRO) and the European Society of Pathology (ESP)) 2020 classification of endometrial carcinoma revised the pathological subtypes and integrated the molecular typing of endometrial cancer [5].

MSI-H endometrial cancer patients are potential beneficiaries of PD-1/PD-L1 inhibitor therapy [6]. MSI is usually as a result of defects in the mismatch repair (MMR) system, a group of enzymes that is responsible for monitoring and repairing the error incorporations in microsatellites [7]. The MMR genes consist of MLH1, MSH2, MSH6 and PMS2. Lynch syndrome results from germline mutations in four MMR genes [8]. Determination of the MSI phenotype in endometrial carcinoma patients by testing MMR status/microsatellite instability (MSI) has been shown to be relevant for four clinical applications: as a surrogate marker for histopathological typing; for Lynch syndrome screening; helping to predict the EC patient's prognosis and determine treatment decisions such as the potential utility of immune checkpoint inhibitor therapy. The International Society of Gynecological Pathology (ISGyP) has suggested universal testing for MMR status/MSI routinely in all endometrial carcinoma cases [9].

There are two assays used to determine MMR-deficient/MSI-H phenotype (simply MSI hereafter) in current clinical testing: MMR—immunohistochemistry (IHC), and PCR-based DNA microsatellite instability analysis (MSI-test) $[9,10]$. MMR-IHC is now the first-line approach to identify patients with MMR deficiency. MMR-IHC is a widely available, costeffective and reliable method to assess MMR status, which can provide direct information on the absence of expression of the altered gene/protein. Alternative MSI-test and subsequent reflex $M L H 1$ hypermethylation, panel testing for germline mutations in $>20$ cancer-causing genes (which include the MMR genes) would be undertaken when indicated.

Identification of the MSI phenotype in colorectal cancer has been well used to help determine treatment decisions for targeted immunotherapy. Some studies also have proven that mismatch-repair deficiency can predict PD-1 blockade response in solid tumors [6,11-13]. Importantly, the UCCN (National Comprehensive Cancer Network) and ESGO/ESTRO/ESP guidelines in 2020 recommended anti-PD- 1 targeted therapy for some advanced MSI EC patients [5]. But its clinical effect remains to be well investigated and improved. Thus, owing to the high prevalence of MMR deficiency in endometrial cancer and potential applications of immunotherapy in EC [1, 14], deeper understanding of MMR-D/MSI-H subtype of endometrial cancer is extremely important.

In the present study, we aimed to explore the relationships between the MSI status and EC clinical features, prognosis, mutation profile, immune infiltrates based on The Cancer Genome Atlas (TCGA) data, and to explore the cell landscape of an MMR-D/MSI-H cancer tissue by single cell-RNA analysis.

\section{Materials and methods}

\subsection{TCGA data collection and analysis}

Gene expression data, somatic mutation profiles, clinical and survival information were downloaded from the TCGA database UCEC project by UCSC Xena [15] (https://xenabrowser.net/datapages/?cohort=GDC\%20TCGA\%20Endometr ioid\%20Cancer\%20(UCEC)\&removeHub=https\%3A\%2F\%2Fxena.treehouse.gi.ucsc.edu\%3A443). RNA expression data were normalized and aligned using R software (version 3.6.2; https://www.r-project.org). Patients whose molecular subtype classification information were unknown were excluded from our study. Thus, data of 500 endometrial cancer patients were analyzed in this study. 


\subsection{Clinicopathological characteristics and prognostic analyses}

We explored the association between molecular subtype and age at diagnosed, BMI by Kruskal-Wallis test. The association between molecular subtype and other clinical variables (clinical stage, histologic grade, diabetes, hypertension, menopause state) were explored by chi-square test. Prognostic analysis of clinical variables was performed for overall survival using Kaplan-Meier curves with log-rank test. For all statistical tests, the $P$ values $<0.05$ were considered statistically significant.

\subsection{Acquisition and analysis of mutation spectra}

The Mutation Annotation Format (MAF) data that were generated by using MuTect2 (4.1.1.0) [16] from whole exome sequencing data were downloaded. The R software (version 3.6.2) "maftools" package was used to provide visual mutation spectra. The Oncoplot function was used to generate waterfall plot of the top 50 mutated genes using COSMIC database.

\subsection{Differentially expressed genes in three subtype groups and enrichment analysis}

The differential analysis was performed by R software (version 3.6.2) "limma" package (version 3.50.0) [17] with one-way analysis of variance (ANOVA). Bonferroni adjusted $\mathrm{P}$ value $<0.05$ was used to filter differentially expressed genes (DEGs). The significant DEGs were visualized using R software "pheatmap" package (version 1.0.12). GO (Gene Ontology) and KEGG (Kyoto Encyclopedia of Genes and Genomes) analysis were performed using R package clusterProfiler (version 4.2.1) [18]. P value $<0.05$ was considered significantly enriched.

\subsection{Estimation of immune composition}

The immune scores were obtained using the ESTIMATE algorithm implemented in R packages estimate (version 1.0.13) [19] based on gene expression data. We compared immune scores across the different molecular subtype groups by Kruskal-Wallis test. We estimated the composition profile of 22 immune cell types of 500 samples using the "Cell type Identification by Estimating Relative Subsets Of RNA Transcripts (CIBERSORT)" algorithm (http://cibersort.stanford.edu/) which included the LM22 signature (PMID: 25822800). Different immune cell infiltration level across the different molecular subtype groups were compared by Kruskal-Wallis test. Overall survival analysis was performed using Kaplan-Meier curves with log-rank test for independent immune cells. For all statistical tests, the $P$ values $<0.05$ were considered statistically significant.

\subsection{Single-cell suspension reparation and $10 \times$ library sequencing}

Following resection, a representative tumor fragment was isolated and transferred rapidly to the laboratory for study as previously described [20]. Briefly, tissue was initially cut into segments and subjected to digestion by collagenase type I/II (Thermo Fisher Scientific, USA) and DNAse I (Sigma, USA). The digested pieces were triturated with a $1 \mathrm{ml}$ syringe plunger and passed through a $70 \mu \mathrm{m}$ cell strainer (Coring, USA). After resuspending in red blood cell lysis buffer (Solarbio, China), live cells were enriched using a Dead Cell Removal kit (Miltenyi Biotec, Germany) as per manufacturer's instructions. Enriched live cells were washed and counted using a hemocytometer with trypan blue. Cells were then resuspended in PBS containing $0.04 \%$ BSA at a concentration of $1 \times 10^{6}$ cells $/ \mathrm{ml}$ with a viability of $>80 \%$ as determined with the Countess. Overall, the entire dissociation procedure took about $2 \mathrm{~h}$ from obtaining sample to generating single-cell suspension. The single-cell suspension was then run on the Chromium 10X device $(10 \times$ Genomics, USA).

Single-cell library preparation was carried out using Chromium Single cell 3' Reagent v2 Kits ( $10 \times$ Genomics, USA) according to the manufacturer's protocol. Then the library was sequenced on the HiSeq X Ten instruments (Illumina, USA) and 150 bp paired-end reads were generated.

\subsection{Single-cell transcriptome data preprocessing and analysis}

We used the Cell Ranger software pipeline (version 2.2.0, 10xGenomics) to process raw sequencing data and Seurat (version 2.3.4) [21] R package for downstream analysis as previously described [20]. Briefly, principle component analysis (PCA) was performed for dimensional reduction. Clusters were identified using the Seurat "FindClusters" algorithm. 
Table 1 Clinical characteristics of 500 patients with endometrial cancer from TCGA database

\begin{tabular}{|c|c|c|c|c|}
\hline & $\begin{array}{l}\text { POLE } \\
(\mathrm{N}=78)\end{array}$ & $\begin{array}{l}\text { MSI } \\
(\mathrm{N}=123)\end{array}$ & $\begin{array}{l}\text { Other } \\
(\mathrm{N}=299)\end{array}$ & $\begin{array}{l}\text { Total } \\
(\mathrm{N}=500)\end{array}$ \\
\hline \multicolumn{5}{|l|}{ Stage } \\
\hline Stage I & $48(61.5 \%)$ & $89(72.4 \%)$ & $172(57.5 \%)$ & 309 (61.8\%) \\
\hline Stage II & $8(10.3 \%)$ & $10(8.1 \%)$ & $30(10.0 \%)$ & $48(9.6 \%)$ \\
\hline Stage III & $19(24.4 \%)$ & $20(16.3 \%)$ & 77 (25.8\%) & $116(23.2 \%)$ \\
\hline Stage IV & $3(3.8 \%)$ & $4(3.3 \%)$ & $20(6.7 \%)$ & $27(5.4 \%)$ \\
\hline \multicolumn{5}{|l|}{ Grade } \\
\hline G1 & $12(15.4 \%)$ & $26(21.1 \%)$ & $55(18.4 \%)$ & $93(18.6 \%)$ \\
\hline $\mathrm{G} 2$ & $14(17.9 \%)$ & $33(26.8 \%)$ & $64(21.4 \%)$ & $111(22.2 \%)$ \\
\hline G3 & $50(64.1 \%)$ & $64(52.0 \%)$ & $173(57.9 \%)$ & $287(57.4 \%)$ \\
\hline High grade & $2(2.6 \%)$ & $0(0 \%)$ & $7(2.3 \%)$ & $9(1.8 \%)$ \\
\hline \multicolumn{5}{|l|}{ Age } \\
\hline$<60$ & $45(57.7 \%)$ & $50(40.7 \%)$ & $72(24.1 \%)$ & 167 (33.4\%) \\
\hline$\geq 60$ & $31(39.7 \%)$ & $73(59.3 \%)$ & $227(75.9 \%)$ & $331(66.2 \%)$ \\
\hline Missing & $2(2.6 \%)$ & $0(0 \%)$ & $0(0 \%)$ & $2(0.4 \%)$ \\
\hline \multicolumn{5}{|l|}{ Radiation therapy } \\
\hline No & $38(48.7 \%)$ & $58(47.2 \%)$ & $164(54.8 \%)$ & $260(52.0 \%)$ \\
\hline Yes & $38(48.7 \%)$ & $54(43.9 \%)$ & $118(39.5 \%)$ & $210(42.0 \%)$ \\
\hline Unknown & $2(2.6 \%)$ & $11(8.9 \%)$ & $17(5.7 \%)$ & $30(6.0 \%)$ \\
\hline \multicolumn{5}{|l|}{ Diabetes } \\
\hline No & $44(56.4 \%)$ & $63(51.2 \%)$ & $143(47.8 \%)$ & $250(50.0 \%)$ \\
\hline Yes & $8(10.3 \%)$ & 21 (17.1\%) & $61(20.4 \%)$ & $90(18.0 \%)$ \\
\hline Unknown & $26(33.3 \%)$ & 39 (31.7\%) & 95 (31.8\%) & $160(32.0 \%)$ \\
\hline \multicolumn{5}{|l|}{ Hypertension } \\
\hline No & $36(46.2 \%)$ & $33(26.8 \%)$ & $83(27.8 \%)$ & $152(30.4 \%)$ \\
\hline Yes & $23(29.5 \%)$ & 54 (43.9\%) & $133(44.5 \%)$ & $210(42.0 \%)$ \\
\hline Unknown & $19(24.4 \%)$ & 36 (29.3\%) & $83(27.8 \%)$ & $138(27.6 \%)$ \\
\hline \multicolumn{5}{|l|}{ Menopause } \\
\hline Pre & $10(12.8 \%)$ & $5(4.1 \%)$ & 17 (5.7\%) & $32(6.4 \%)$ \\
\hline Peri & $6(7.7 \%)$ & $4(3.3 \%)$ & $7(2.3 \%)$ & $17(3.4 \%)$ \\
\hline Post & $51(65.4 \%)$ & $106(86.2 \%)$ & $252(84.3 \%)$ & 409 (81.8\%) \\
\hline Indeterminate & $2(2.6 \%)$ & $5(4.1 \%)$ & $8(2.7 \%)$ & $15(3.0 \%)$ \\
\hline Unknown & $9(11.5 \%)$ & $3(2.4 \%)$ & $15(5.0 \%)$ & 27 (5.4\%) \\
\hline \multicolumn{5}{|l|}{ BMI } \\
\hline$<25$ & $24(30.8 \%)$ & $17(13.8 \%)$ & $43(14.4 \%)$ & $84(16.8 \%)$ \\
\hline$\geq 25$ & $47(60.3 \%)$ & 102 (82.9\%) & $238(79.6 \%)$ & 387 (77.4\%) \\
\hline Missing & 7 (9.0\%) & 4 (3.3\%) & $18(6.0 \%)$ & 29 (5.8\%) \\
\hline
\end{tabular}

Graph-based clustering results on 20 principle components were visualized in 2-dimension using t-SNE. Cell clusters were annotated to known biological cell types using canonical marker genes. A cluster-specific biomarker was found by "FindAllMarkers" function identified when it was expressed in a minimum of $25 \%$ of cells and at a minimum log fold change threshold of 0.25 .

\subsection{Immunohistochemistry}

Tissue sections were collected and fixed in 10\% formalin. $5 \mu \mathrm{M}$ slides were used for immunohistochemistry analysis. The "UltraVision Quanto Detection System HRP DAB" IHC kit (TL-125-QDH, Thermo Fisher Scientific) was used for the tyramide signal amplification according to the manufacturer's protocol. Primary antibodies used in this assay are as follows: anti-CD20 (M0755, Dako), anti-MLH1 (MAB-0789, MXB Biotechnologies), anti-MSH2 (MAB-0836, MXB Biotechnologies), anti-PMS2 (GT215902, Gene Tech) and anti-MSH6 (MAB-0831, MXB Biotechnologies). Images were taken and quantitative 
A
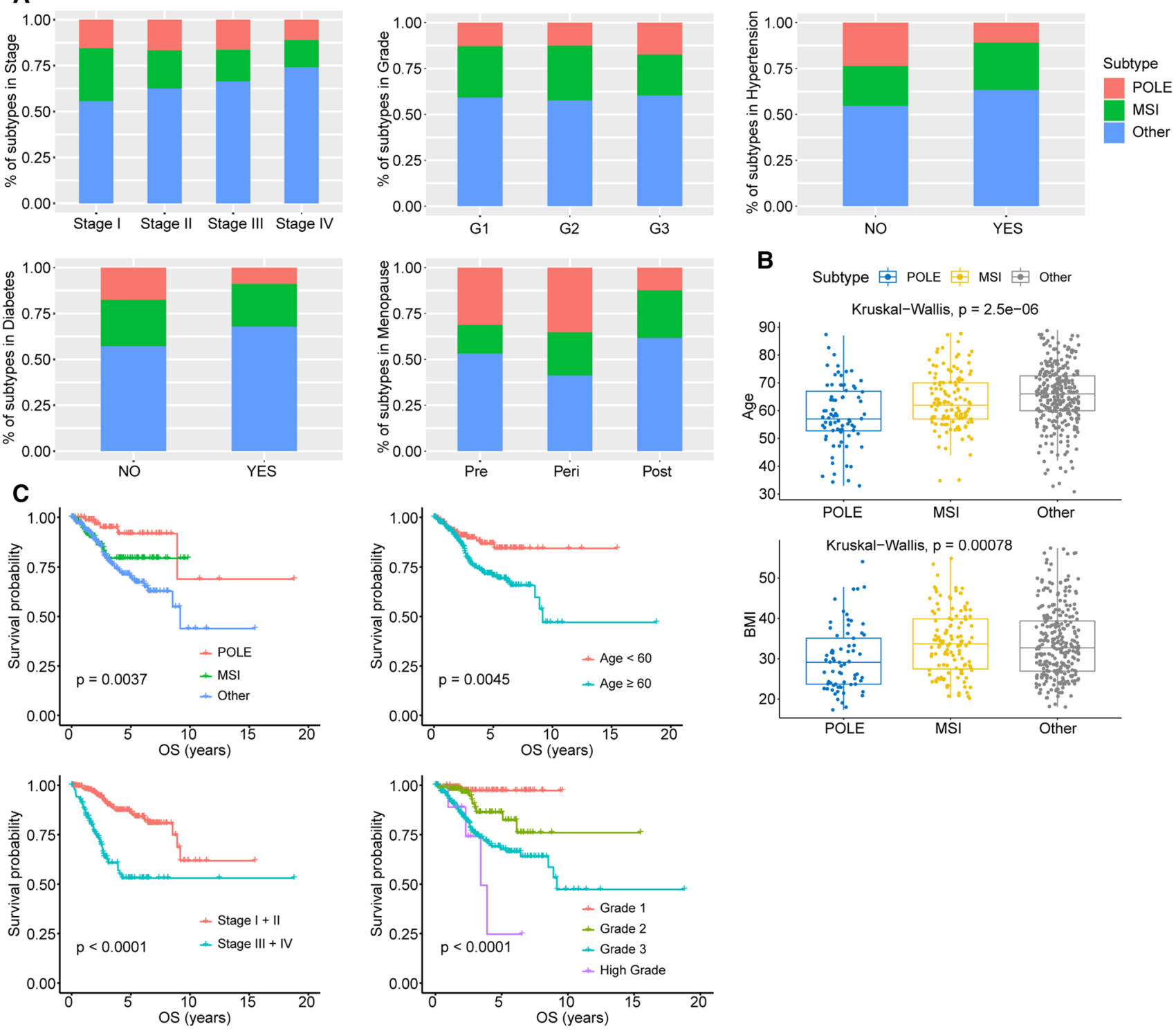

Fig. 1 Association between molecular subtypes and clinical variables and survival outcome. A The fractions of molecular subtypes in clinical stage, histologic grade, diabetes, hypertension and menopause state. Chi-square test, $\mathrm{P}$ values $<0.05$ were considered statistically significant. B Distribution of age and BMI of EC molecular subtypes. Kruskal-Wallis test, P values $<0.05$ were considered statistically significant. C Overall survival curve and log-rank test for endometrial cancer patients based on molecular subtype, clinical stage, histologic grade and age classification. $P$ values $<0.05$ were considered statistically significant

image analysis was performed using ImagePro software. For comparison for CD20 between two groups, statistical evaluation was done by two-tailed Student's t-test, error bars show standard error of the mean (SEM).

\subsection{Statistical analyses}

Statistical analyses were performed using GraphPad Prism 5 software and statistical significance was determined by $\mathrm{p}<0.05$. For TCGA mutation and RNA-seq data, all statistical analysis was performed with $\mathrm{R}$ (version 3.6.2). 

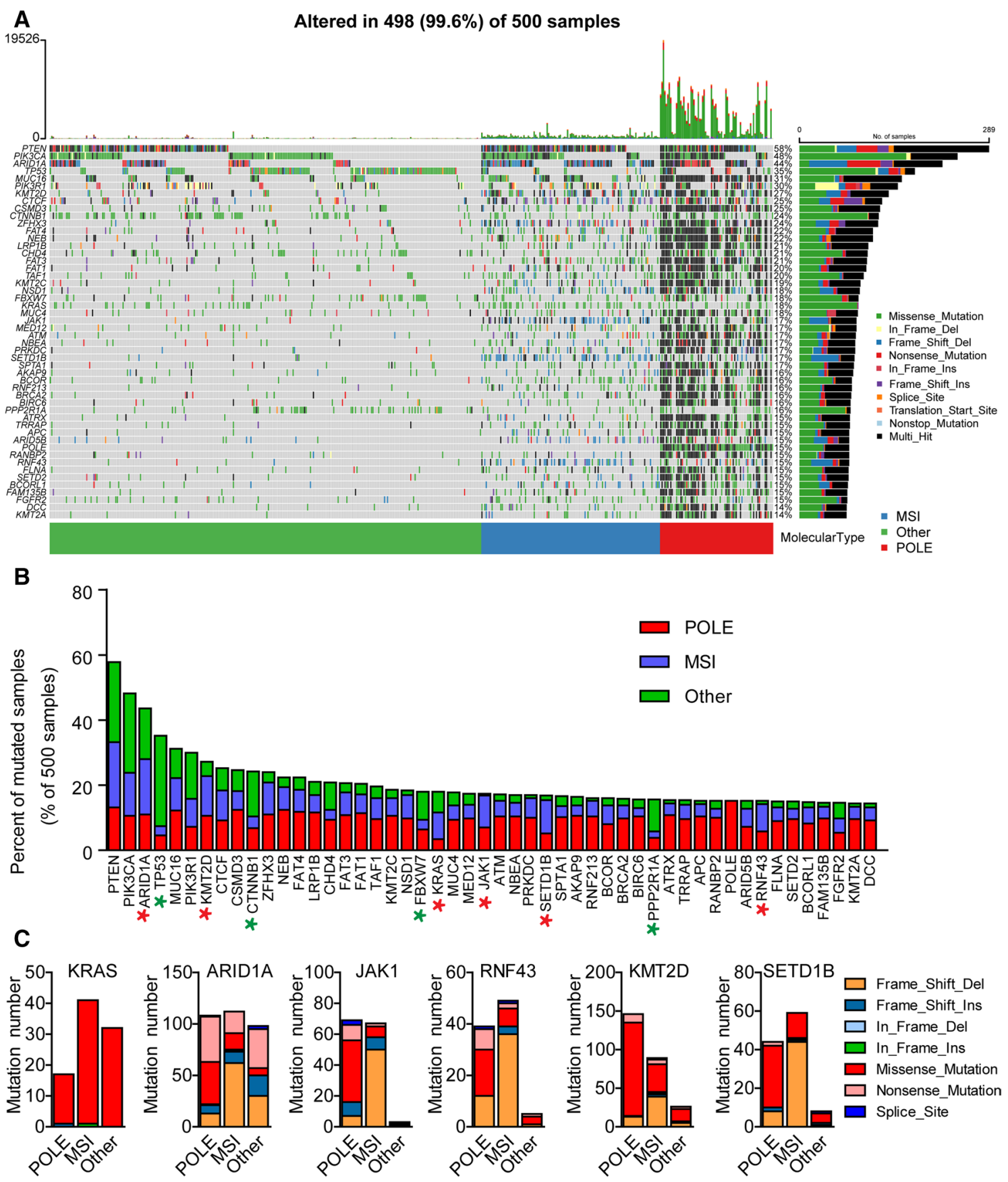

Fig. 2 Landscape of somatic mutation profiles in 500 endometrial cancer samples from TCGA database. AThe waterfall plot shows top 50 mutated genes in each sample. The upper barplot shows the number of mutations in each patient, while the right barplot indicates the number of mutated samples in each gene. B Proportion of tumors in three molecular subtypes of each gene. Green stars showed 4 genes with few mutations in MSI group, red stars showed 6 genes with frequent mutations in MSI group. C Frequently mutated genes in the MSI subgroup. Shown are the mutation numbers of different variant types in six genes

\section{Results}

\subsection{Association between EC molecular subtypes and survival and clinical features}

To explore the relationship between the distinct molecular subtypes of endometrial cancer and the clinical features, we analyzed clinical data of 500 EC patients from TCGA database, including clinical stage, histologic grade, age, history of 
Fig. 3 Enrichment analysis of up-regulated genes (DEGs) in POLE and MSI groups compared with Other ECs. A, B The top 10 of biological processes GO terms (A) and KEGG (Kyoto Encyclopedia of Genes and Genomes) pathway (B) enriched by 1138 genes those were upregulated in POLE and MSI groups compared with Other ECs. P value $<0.05$ was considered significantly enriched

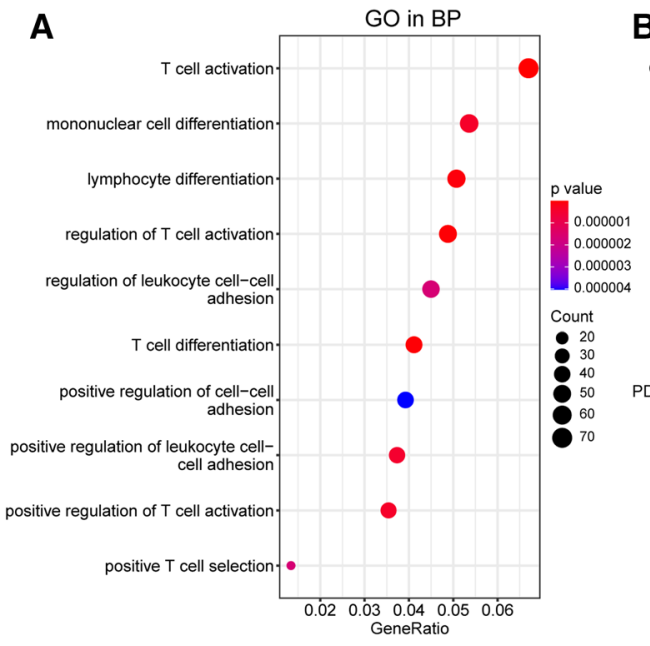

radiation therapy, diabetes, hypertension, menopause state and BMI (Table 1). The 500 patients were classified into three molecular subgroups: the POLE, MSI and Other subgroup. We discovered that the POLE subgroup has a lower age, BMI, lower fraction of diabetes and hypertension (Fig. 1A, B). The tumors in MSI subgroup were identified more often in the early stage, and had a lower age than the Other subgroup (Fig. 1A, B). These results were consistent with the prognosis analysis between patient survival and molecular subtype, age and clinical stage (Fig. 1A-C). But there is no difference in the proportion of histologic grade across the subtypes (Fig. 1A). The POLE and MSI subtypes, lower age, grade and clinical stage were all correlated with better patient survival (Fig. 1C).

\subsection{Mutation spectra in POLE, MSI, and Other ECs}

Tumors with POLE mutation or MSI have been suggested to be hyper or highly mutated. Mutation spectra across 500 EC patients in the TCGA database were analyzed. Most of the genes had higher mutation frequency in the POLE group (Fig. 2A). The MSI group had few mutations in TP53, FBXW7, CTNNB1 and PPP2R1A, which was consistent with previously described (Fig. 2B) [4]. 6 genes showed more frequent mutations in MSI group, including 4 genes (KRAS, ARID1A, JAK1 and RNF43) $[4,22]$ that have been previously reported in endometrial cancer and 2 novel genes (KMT2D and SETD1B) (Fig. 2B). Except for KRAS with predominantly missense mutation in all groups, other 5 genes showed more frequent frameshift deletions in MSI group than POLE and other groups (Fig. 2C). JAK1 and RNF43 with polymerase slippageassociated deletions have been reported previously [22]. KMT2D and SETD1B are chromatin remodeling-related genes, and appear to help predict the degree of myometrial invasion [23].

\subsection{Enriched immune infiltrates in POLE and MSI ECs than Other ECs}

We analyzed the global gene expression profile of $500 \mathrm{EC}$ patients, and identified the differentially expressed genes (DEGs) between the three molecular groups. Differential expression analysis showed that 1138 genes were significantly upregulated in MSI and POLE groups compared with other group (Supplementary table 1, Figure S1). The gene set enrichment analyses (GSEA) using Gene Ontology (GO) terms and Kyoto Encyclopedia of Genes and Genomes (KEGG) terms showed that genes up-regulated in POLE and MSI samples were mainly enriched for immune-related functions, such as T cell activation, T cell differentiation, cytokine-cytokine receptor interaction and T cell receptor signaling pathway (Fig. 3).

POLE mutation or MSI subtypes in cancers indicate hypermutations, and they are suggested to harbor more tumorspecific neoantigens and higher lymphocytes infiltrates [24]. Thus, we calculated the immune scores of all 500 samples using the ESTIMATE algorithm, and found that immune scores of both POLE and MSI EC groups were significantly higher than the Other group (Fig. 4A). To determine the correlation between molecular subtypes and immune composition, we then estimated the composition profile of 22 immune cell types of 500 samples using CIBERSORT algorithm (Fig. 4B). Furthermore, the Kaplan-Meier curve with log-rank test was used to analyze the correlation between each immune infiltrate level and the overall survival (OS) time of EC patients. The fractions of $10 \mathrm{immune}$ cell types varied across molecular subtypes (Fig. 4C). Importantly, both POLE and MSI groups were found to have more CD8 ${ }^{+} \mathrm{T}$ cells, follicular helper T cells, resting NK cells, M1 macrophages, while less activated NK cells, M2 macrophages, activated dendritic cells and mast cells 

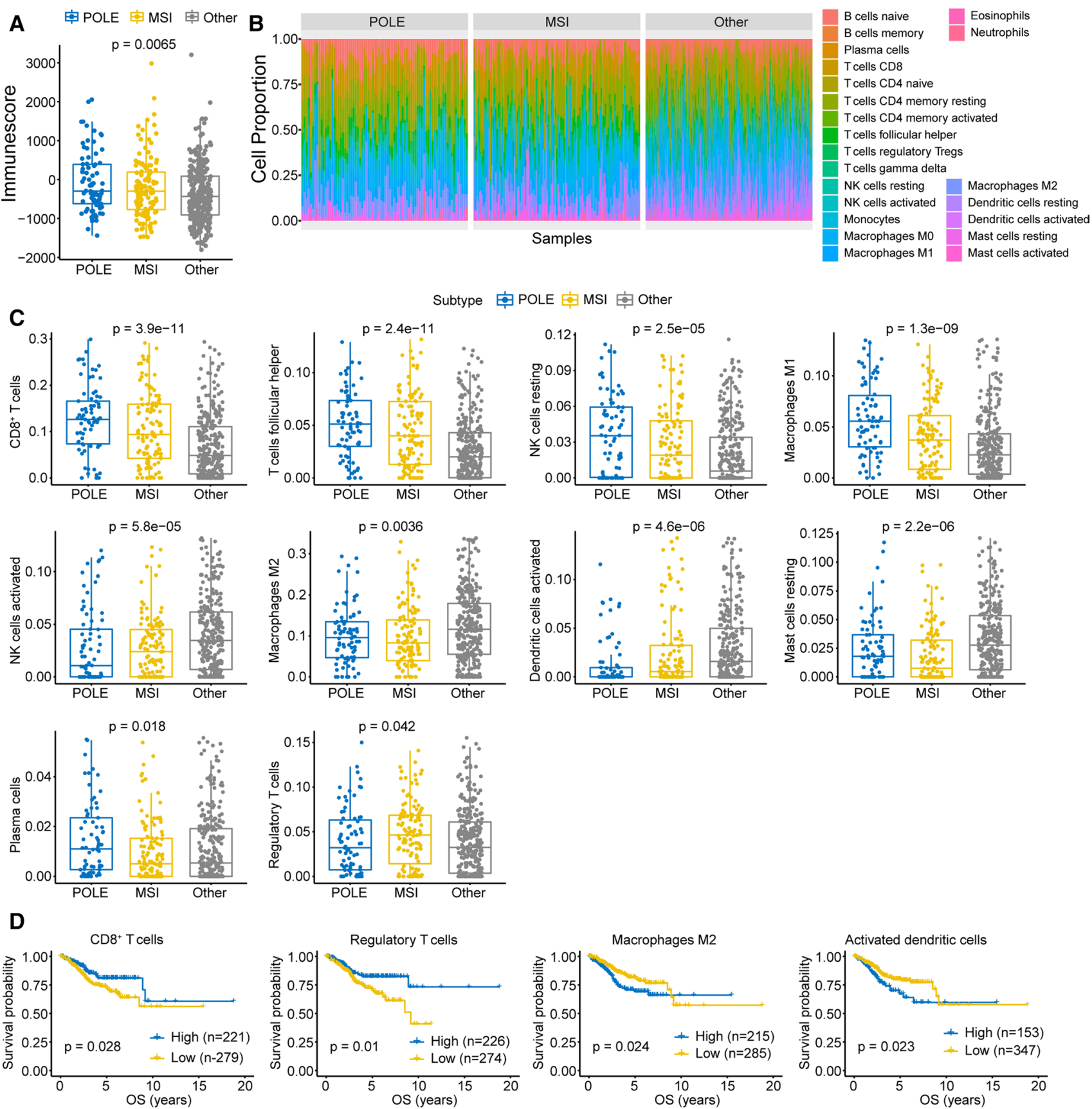

Fig. 4 Immune infiltrates in EC samples from TCGA database. A Distribution of immune scores of EC molecular type, including POLE, MSI, and Other type. Kruskal-Wallis test. The P values $<0.05$ were considered statistically significant. B The fractions of 22 immune cell types in 500 EC samples using CIBERSORT algorithm. C Relationship between different immune cell types and EC molecular type. Distribution of $\mathrm{CD}^{+} \mathrm{T}$ cells, follicular helper T cells, resting NK cells, M1 macrophages, activated NK cells, M2 macrophages, activated dendritic cells, mast cells, plasma cells and regulatory T cells across EC molecular type. Kruskal-Wallis test. The P values $<0.05$ were considered statistically significant. D Kaplan-Meier curves with log-rank test for two immune cell types associated with good prognosis and two types associated with poor prognosis. The $\mathrm{P}$ values $<0.05$ were considered statistically significant

(Fig. 4C). POLE groups had more plasma cells (Fig. 4C). MSI group had more regulatory T cells (Fig. 4C). Among these immune cell types, high infiltrates level of $\mathrm{CD} 8^{+} \mathrm{T}$ cells, and regulatory $\mathrm{T}$ cells were found to be significantly positively related with OS, while high infiltrates level of $\mathrm{M} 2$ macrophages and activated dendritic cells were negatively related with OS (Fig. 4D). These results were consistent with better clinical outcomes in POLE and MSI EC groups than Other group. 


\subsection{Cell mapping of immune ecosystem in an MMR-D EC sample}

Our previous study also indicated higher lymphocytes infiltration in an MMR-D cancer tissue compared to other (MMR-I) samples [20]. To gain deeper insight into the immune cellular composition and phenotypic diversity of MMR-D endometrial cancer, we profiled 3371 cells obtained from an MMR-D EC sample (labeled "EC-MSI") using the single-cell RNA-seq (10X Genomics platform). The immunohistochemistry (IHC) staining of the mismatch repair (MMR) proteins showed that MSH2 and MSH6 expression were negative (Fig. 5A). The single-cell RNA-seq data was subjected to quality filtering and downstream analysis using Seurat R (version 2.3.4) package. Graph-based clustering (Fig. 5B) was used to classify cells into groups. We identified 4 major immune cell types through marker genes: proliferative immune cells, T cells, B cells, myeloid cells in this sample (Fig. 5C). The immune cells were further analyzed by identifying their subsets. T cells included conventional $C D 4^{+} T$ cells (cluster $0 ; C D 4^{+}$), regulatory $T$ cells (cluster $7 ; F O X P 3^{+}$), $C D 8^{+} T$ cells (cluster 3,6 and $10 ; C D 8 B^{+}$), exhausted T cells (cluster 2; $P D C D 1^{+}$) and natural killer T cells (NKT cells) (cluster 9; $G N L Y^{+}$) (Fig. 5C, D). Proliferative immune cells $\left(M K I 67^{+}\right)$consisted of both T and B cell lineages, including: proliferative $C D 4^{+} T$ cells (cluster 11$)$, proliferative $C D 8^{+}$ T cells (cluster 13), proliferative follicular B cells (cluster 12) and proliferative plasma B cells (cluster 5) (Fig. 5 C and E). Two clusters (cluster 1 and 14) of myeloid cells were characterized by enriched expression of CD68 (Fig. 5F). Two subtypes in $B$ cells were further analyzed. Follicular B cells were (cluster 8 ) enriched for the expression of CD19 and MS4A1 [25], while plasma B cells (cluster 4) were characterized by the expression of MZB1 (Fig. 5C, G) [26]. We also reanalyzed the single-cell RNA-seq data of another MMR-D sample (EC5-T) published before [20] and decoded the immune ecosystem (Figure S2). We found that the two samples showed similar immune phenotypic diversity (Figure S2). These results formed the basis description of the immune subsets in endometrial carcinomas with MSI.

Brooke E et al. reported that the MSI tumors exhibited higher infiltration of $\mathrm{CD}^{+}$and $\mathrm{CD}^{+}{ }^{+}$cells compared to MSS tumors [27]. However, little is known about the relationship between MSI status and tumor infiltrating B cells. Here, we assessed the infiltration of B cells by IHC staining of MMR-D and MMR-I tumor sections with CD20 antibody (Fig. $5 \mathrm{H}$ ). The MMR-D tumors exhibited a higher trend in the number of B cells than MMR-I tumors, but with no significance (Fig. 5I). Patient overall survival analysis from TCGA-UCEC data indicated that higher expression of CD20 (MS4A1) was associated with good prognosis (Fig. $5 \mathrm{~J}$ ). The function of B cells in endometrial carcinomas remains to be further explored.

\section{Discussion}

Distinct factors affect the prognosis of endometrial cancer such as tumor type, grade and stage status [28, 29]. MMR-D subtype has been reported to be related with a good prognosis in endometrial cancer patients [14]. Moreover, promising clinical trials of immunotherapy (anti-PD-1/anti-PD-L1) indicate good response in advanced MMR-D/MSI-H EC patients $[30,31]$. But the underlying mechanism between the MMR-D status and endometrial cancer patient prognosis is not well investigated and understood. In this study, we related the molecular subtype to clinical data and prognosis of EC patients based on 78 POLE, $123 \mathrm{MSI}$ and 299 Other EC samples in the TCGA-UCEC project. The tumors in MSI subgroup were identified more often in early stage, had a lower age, better patient survival and enriched immune infiltrates than the Other subgroup. Besides, using the unbiased single-cell RNA-seq analysis for an MMR-D endometrial cancer tissue, an immune atlas was established.

Tumors with defects in MMR proteins indicated higher mutation frequency, and were suggested to harbor more tumor-specific neoantigens and higher lymphocytes infiltrates [24]. Here, our enrichment analysis of up-regulated genes in POLE and MSI subtype also suggested the enrichment of immune-related functions. Diversity of tumor infiltrating immune cells in tumor influences tumor development, progression, and treatment response to targeted agents. CD8 ${ }^{+}$

\section{(See fig on next page.)}

Fig. 5 Atlas of multiple immune cell types in an MMR-D endometrial carcinoma sample (EC-MSI) and enrichment trend of B cells in MMR-D endometrial tumors. A IHC staining images of MLH1, MSH2, MSH6 and PMS2 in tumor slides isolated from the MMR-D sample. Scale bars, $60 \mu \mathrm{m}$. B t-SNE projection of all cells, color-coded by their associated cluster, and labeled with cluster number. C Cell cluster, number and marker genes of annotated immune cell types are summarized. D-G t-SNE map, colored by relative expression (lowest expression to highest expression, gray to red) of marker genes for immune cell subtypes: T cells (D), proliferative immune cells (E), myeloid cells (F), and B cells (G). H Representative IHC staining images of CD20 in slides isolated from MMR-D and MMR-I endometrial carcinoma sections. Scale bars, $60 \mu \mathrm{m}$. I Quantification of the numbers of B cells as presented in (B). Data are means \pm SEM (15 MMR-D sections and 15 MMR-I sections were analyzed). Student's t-test. J The overall survival curves with log-rank test for MS4A1 (CD20) expression based on TCGA-UCEC data. P value $<0.05$ were considered statistically significant 

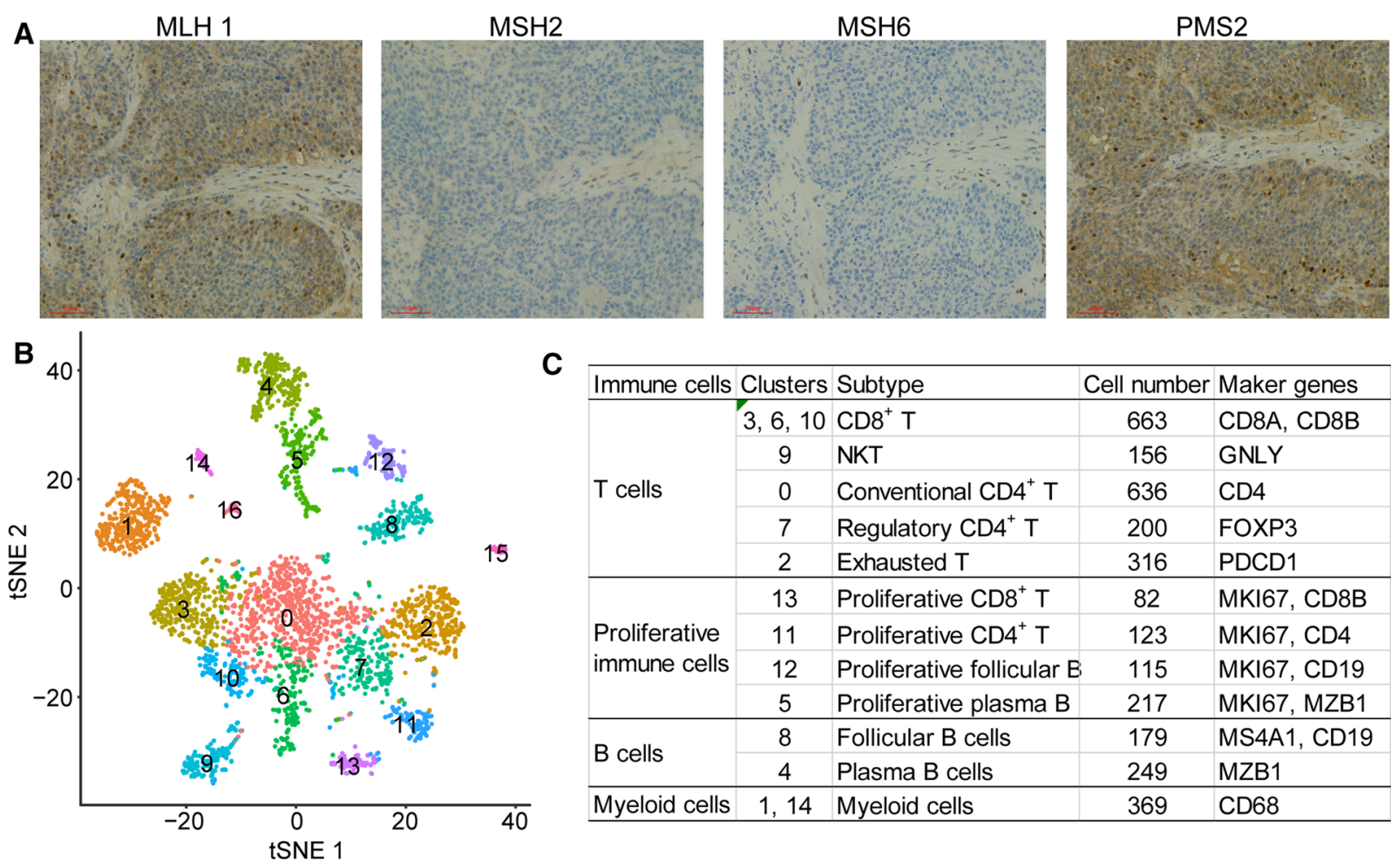

C

\begin{tabular}{|c|c|c|c|c|}
\hline Immune cells & Clusters & Subtype & Cell number & Maker genes \\
\hline \multirow{5}{*}{ T cells } & $3,6,10$ & $\mathrm{CD}^{+} \mathrm{T}$ & 663 & CD8A, CD8B \\
\hline & 9 & NKT & 156 & GNLY \\
\hline & 0 & Conventional $\mathrm{CD}^{+} \mathrm{T}$ & 636 & CD4 \\
\hline & 7 & Regulatory $\mathrm{CD}^{+} \mathrm{T}$ & 200 & FOXP3 \\
\hline & 2 & Exhausted T & 316 & PDCD1 \\
\hline \multirow{4}{*}{$\begin{array}{l}\text { Proliferative } \\
\text { immune cells }\end{array}$} & 13 & Proliferative $\mathrm{CD}^{+} \mathrm{T}$ & 82 & MKI67, CD8B \\
\hline & 11 & Proliferative $\mathrm{CD} 4^{+} \mathrm{T}$ & 123 & MKI67, CD4 \\
\hline & 12 & Proliferative follicular B & 115 & MKI67, CD19 \\
\hline & 5 & Proliferative plasma B & 217 & MKI67, MZB1 \\
\hline \multirow{2}{*}{ B cells } & 8 & Follicular B cells & 179 & MS4A1, CD19 \\
\hline & 4 & Plasma B cells & 249 & MZB1 \\
\hline Myeloid cells & 1,14 & Myeloid cells & 369 & CD68 \\
\hline
\end{tabular}

$\begin{array}{lll}\text { D } & \mathrm{CD} 8^{+} \mathrm{T} & \mathrm{NKT}\end{array}$

Conventional $\mathrm{CD}^{+} \mathrm{T} \quad$ Regulatory $\mathrm{CD} 4^{+} \mathrm{T}$

Exhausted T
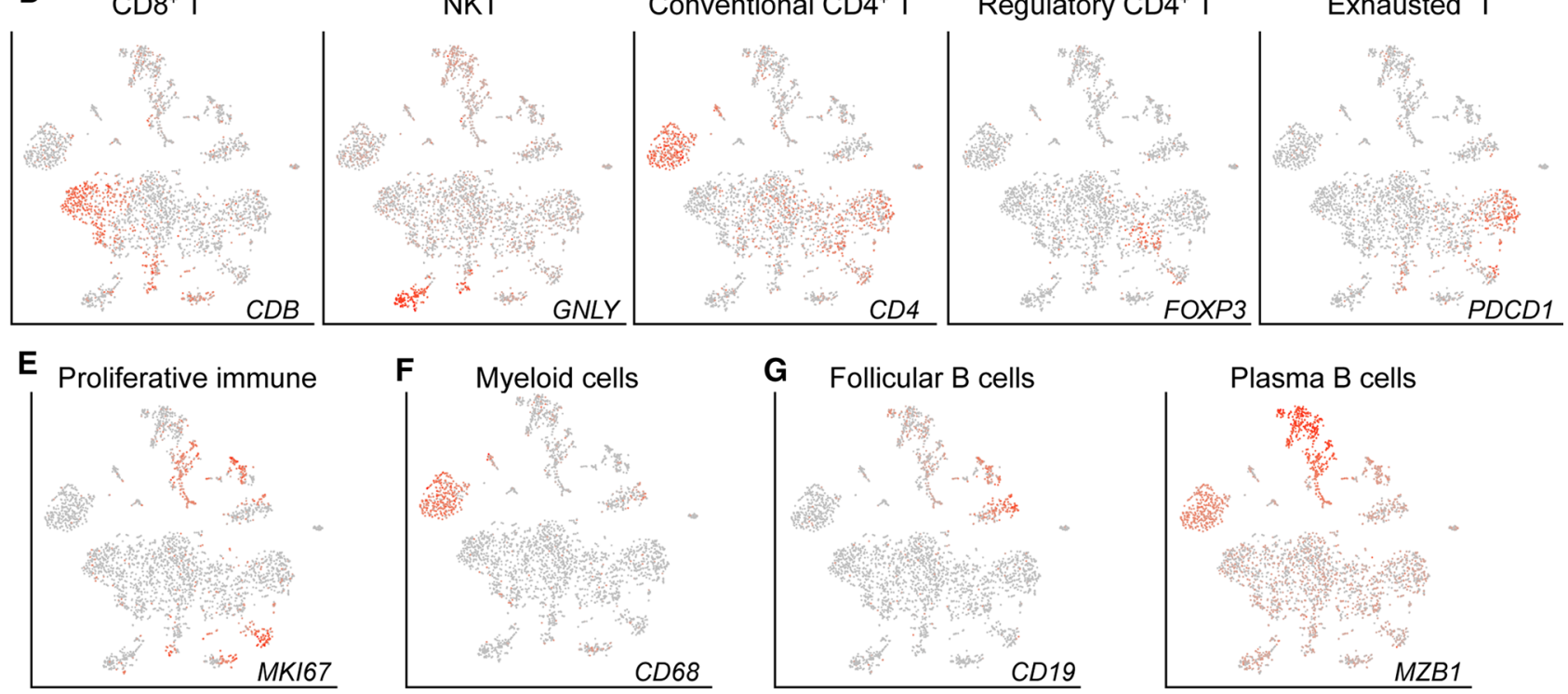

G Follicular B cells

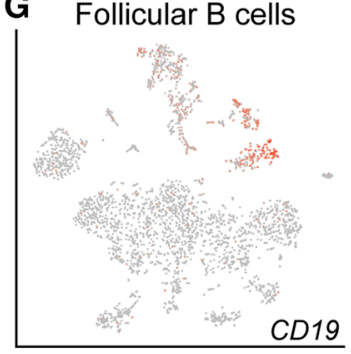

Plasma B cells

H

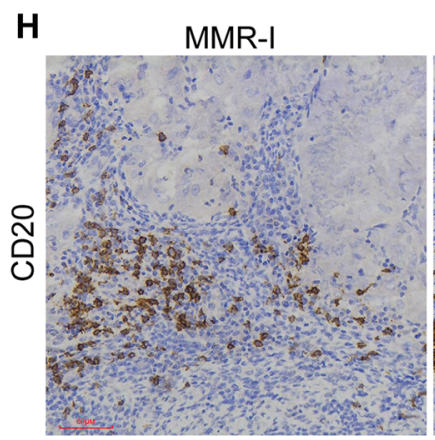

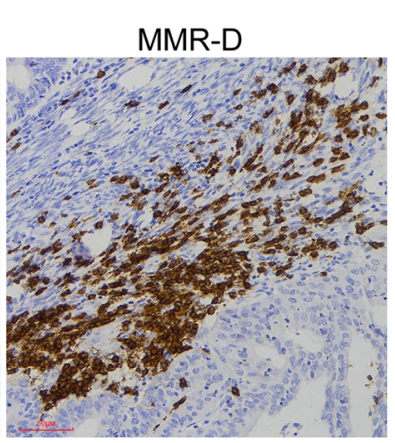

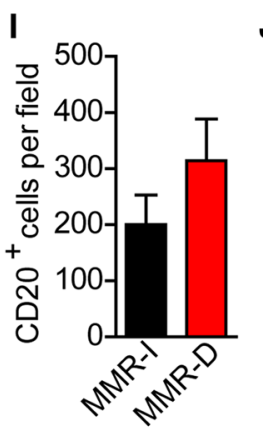

J

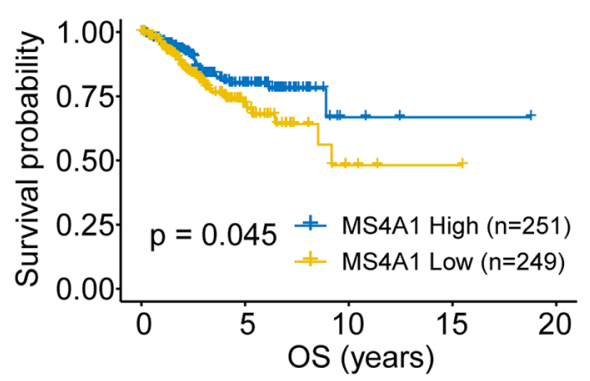


T cells are essential for successful tumor killing. Regulatory T cells suppress anti-tumor immune response, and are usually associated with poor clinical outcomes [32]. A previous study reported that tumor-associated macrophages (TAMs) revealed a pro-tumor role in endometrial cancer [33]. Dendritic cells could be modulated by tumor cells, and thus drive immune tolerance [34]. In this study, both $\mathrm{CD}^{+} \mathrm{T}$ cells and regulatory T cells showed high infiltration in MSI subtype and were related with good OS. M2 macrophages and activated dendritic cells were found to be negatively correlated with MSI subtype and patient prognosis. The high infiltration of regulatory $T$ cells might result from high neoantigens. Further investigations are necessary to clarify the exact functions of regulatory T cells and activated dendritic cells in EC.

B cells are the other major type of tumor-infiltrating lymphocytes (TILs) besides T cells. Several studies unveil that B cells suppress the progression of tumor. B cell depletion enhanced melanoma growth in mice [35]. Activation of B cells play an anti-tumor role by secreting immunoglobulins, promoting T cell response, and killing cancer cells directly [36, 37]. In this study, we tested MMR status of 98 EC patients, and identified 15 MMR-D cases. By comparing the infiltration of $C D 20^{+} B$ cells between 15 pairs of MMR-D and MMR-I EC tumors, we found that the MMR-D tumors showed a higher trend in the number of $C D 20^{+} B$ cells and that higher expression of CD20 (MS4A1) was associated with good prognosis. Two major subtypes of B cells and their versatile functions in non-small cell lung cancer have been reported [38]. Studies on $B$ cell subtypes in EC are sparse, and the subsets and functions of $B$ cells in the tumor microenvironment (TME) of EC remain largely unknown. We found two subtypes of $B$ cells (follicular $B$ cells and plasma B cells) in MMR-D EC, while the functions of different $B$ cell subtypes necessitating further investigations to answer.

Tumors are complex ecosystems characterized by different compositions and functions of tumor-infiltrating immune cells and other stromal cells [39]. The TME characteristics plays a critical role in the responsiveness of immunotherapy [40]. Single-cell RNA-seq technology allowed for the in-depth profiling of heterogeneous immune cell populations. In this study, we provided a baseline description of the immune transcriptomes by single-cell RNA-seq analysis for an MMR-D EC tumor, which formed the basis for further examination into the role of immune subsets in endometrial carcinomas. Future studies are needed to determine the specific roles these complex immune cell types play in the regulation of EC development and progression.

Authors' contributions Y-eG and YL conceived this study. Y-eG and YL performed experiments and data analysis. Y-eG and YL jointly wrote this manuscript. GC and YL supervised this work. HL, WZ, and PS participated in the discussion. All authors have read and approved the final manuscript.

Funding This work was supported by grants from the National Natural Science Foundation of China (Grant 81802960 to Guofang Chen and Grant 31801111 to Yin Liu), and Dream Mentor-Outstanding Young Talents Program (Grant fkyq1910 to Yin Liu).

Data availability The single-cell sequencing raw data and processed data of EC-MSI have been deposited into the NCBI GEO database: GSE193430. Single-cell RNA-seq data of our published MMR-D sample (EC5-T) in PRJNA650549 were downloaded from the SRA database (https://www.ncbi.nlm.nih.gov/sra/PRJNA650549). All other data can be provided upon reasonable request to the corresponding author.

Code availability The code used in the current study are available from the corresponding author on reasonable request.

\section{Declarations}

Competing interests The authors declare no competing interests.

Open Access This article is licensed under a Creative Commons Attribution 4.0 International License, which permits use, sharing, adaptation, distribution and reproduction in any medium or format, as long as you give appropriate credit to the original author(s) and the source, provide a link to the Creative Commons licence, and indicate if changes were made. The images or other third party material in this article are included in the article's Creative Commons licence, unless indicated otherwise in a credit line to the material. If material is not included in the article's Creative Commons licence and your intended use is not permitted by statutory regulation or exceeds the permitted use, you will need to obtain permission directly from the copyright holder. To view a copy of this licence, visit http://creativecommons.org/licenses/by/4.0/.

\section{References}

1. Morice $P$, et al. Endometrial cancer. Lancet. 2016;387(10023):1094-108.

2. Di Cristofano A, Ellenson LH. Endometrial carcinoma. Annu Rev Pathol. 2007;2:57-85.

3. Bell DW, Ellenson LH. Molecular genetics of endometrial carcinoma. Annu Rev Pathol. 2019;14:339-67.

4. Cancer Genome Atlas Research, et al. Integrated genomic characterization of endometrial carcinoma. Nature. 2013;497(7447):67-73. 
5. Concin N, et al. ESGO/ESTRO/ESP guidelines for the management of patients with endometrial carcinoma. Int J Gynecol Cancer. 2021;31(1):12-39.

6. Le DT, et al. Mismatch repair deficiency predicts response of solid tumors to PD-1 blockade. Science. 2017;357(6349):409-13.

7. Laghi L, Bianchi P, Malesci A. Differences and evolution of the methods for the assessment of microsatellite instability. Oncogene. 2008;27(49):6313-21.

8. Hampel $\mathrm{H}$, et al. Screening for Lynch syndrome (hereditary nonpolyposis colorectal cancer) among endometrial cancer patients. Cancer Res. 2006;66(15):7810-7.

9. Cho KR, et al. International Society of Gynecological Pathologists (ISGyP) Endometrial Cancer Project: Guidelines From the Special Techniques and Ancillary Studies Group. Int J Gynecol Pathol. 2019;38(Suppl 1):S114-22.

10. Zheng J, et al. The clinicopathological features and prognosis of tumor MSI in East Asian colorectal cancer patients using NCl panel. Future Oncol. 2018;14(14):1355-64.

11. Asaoka Y, ljichi H, Koike K. PD-1 blockade in tumors with mismatch-repair deficiency. N Engl J Med. 2015;373(20):1979.

12. Diaz LA, Le DT. PD-1 blockade in tumors with mismatch-repair deficiency. N Engl J Med. 2015;373(20):1979.

13. Le DT, et al. PD-1 blockade in tumors with mismatch-repair deficiency. N Engl J Med. 2015;372(26):2509-20.

14. Haruma T, et al. Clinical impact of endometrial cancer stratified by genetic mutational profiles, POLE mutation, and microsatellite instability. PLoS ONE. 2018;13(4): e0195655.

15. Goldman MJ, et al. Visualizing and interpreting cancer genomics data via the Xena platform. Nat Biotechnol. 2020;38(6):675-8.

16. Cibulskis $\mathrm{K}$, et al. Sensitive detection of somatic point mutations in impure and heterogeneous cancer samples. Nat Biotechnol. 2013;31(3):213-9.

17. Ritchie ME, et al. limma powers differential expression analyses for RNA-sequencing and microarray studies. Nucleic Acids Res. 2015;43(7): e47.

18. Wu T, et al. clusterProfiler 4.0: a universal enrichment tool for interpreting omics data. Innovation. 2021;2(3): 100141.

19. Yoshihara K, et al. Inferring tumour purity and stromal and immune cell admixture from expression data. Nat Commun. 2013;4:2612.

20. Guo YE, et al. Phenotyping of immune and endometrial epithelial cells in endometrial carcinomas revealed by single-cell RNA sequencing. Aging (Albany NY). 2021;13(5):6565-91.

21. Stuart T, et al. Comprehensive integration of single-cell data. Cell. 2019;177(7):1888-902.

22. Gibson WJ, et al. The genomic landscape and evolution of endometrial carcinoma progression and abdominopelvic metastasis. Nat Genet. 2016;48(8):848-55.

23. Garcia-Sanz $\mathrm{P}$, et al. Chromatin remodelling and DNA repair genes are frequently mutated in endometrioid endometrial carcinoma. Int J Cancer. 2017;140(7):1551-63.

24. Howitt $B E$, et al. Association of polymerase e-mutated and microsatellite-instable endometrial cancers with neoantigen load, number of tumor-infiltrating lymphocytes, and expression of PD-1 and PD-L1. JAMA Oncol. 2015;1(9):1319-23.

25. Lambrechts $D$, et al. Phenotype molding of stromal cells in the lung tumor microenvironment. Nat Med. 2018;24(8):1277-89.

26. MacParland SA, et al. Single cell RNA sequencing of human liver reveals distinct intrahepatic macrophage populations. Nat Commun. 2018;9(1):4383.

27. van Gool IC, et al. POLE proofreading mutations elicit an antitumor immune response in endometrial cancer. Clin Cancer Res. 2015;21(14):3347-55.

28. Steiner $E$, et al. Multivariate independent prognostic factors in endometrial carcinoma: a clinicopathologic study in 181 patients: 10 years experience at the Department of Obstetrics and Gynecology of the Mainz University. Int J Gynecol Cancer. 2003;13(2):197-203.

29. Li Y, et al. POLE mutations improve the prognosis of endometrial cancer via regulating cellular metabolism through AMF/AMFR signal transduction. BMC Med Genet. 2019;20(1):202.

30. Marabelle A, et al. Efficacy of pembrolizumab in patients with noncolorectal high microsatellite instability/mismatch repair-deficient cancer: results from the phase II KEYNOTE-158 study. J Clin Oncol. 2020;38(1):1-10.

31. Makker V, et al. Lenvatinib plus pembrolizumab in patients with advanced endometrial cancer: an interim analysis of a multicentre, openlabel, single-arm, phase 2 trial. Lancet Oncol. 2019;20(5):711-8.

32. Tanaka A, Sakaguchi S. Regulatory T cells in cancer immunotherapy. Cell Res. 2017;27(1):109-18.

33. Tong $\mathrm{H}$, et al. Tumor-associated macrophage-derived CXCL8 could induce ERalpha suppression via HOXB13 in endometrial cancer. Cancer Lett. 2016;376(1):127-36.

34. Wculek SK, et al. Dendritic cells in cancer immunology and immunotherapy. Nat Rev Immunol. 2020;20(1):7-24.

35. DiLillo DJ, Yanaba K, Tedder TF. B cells are required for optimal CD4+ and CD8+ T cell tumor immunity: therapeutic B cell depletion enhances B16 melanoma growth in mice. J Immunol. 2010;184(7):4006-16.

36. Tokunaga R, et al. B cell and B cell-related pathways for novel cancer treatments. Cancer Treat Rev. 2019;73:10-9.

37. Li Q, et al. In vivo sensitized and in vitro activated B cells mediate tumor regression in cancer adoptive immunotherapy. J Immunol. 2009;183(5):3195-203.

38. Chen J, et al. Single-cell transcriptome and antigen-immunoglobin analysis reveals the diversity of B cells in non-small cell lung cancer. Genome Biol. 2020;21(1):152.

39. Klemm F, Joyce JA. Microenvironmental regulation of therapeutic response in cancer. Trends Cell Biol. 2015;25(4):198-213.

40. McMillin DW, Negri JM, Mitsiades CS. The role of tumour-stromal interactions in modifying drug response: challenges and opportunities. Nat Rev Drug Discov. 2013;12(3):217-28.

Publisher's Note Springer Nature remains neutral with regard to jurisdictional claims in published maps and institutional affiliations. 